Editorial

\title{
Aerospace Best Paper Awards 2017
}

\author{
Aerospace Editorial Office
}

MDPI, St. Alban-Anlage 66, 4052 Basel, Switzerland; aerospace@mdpi.com

Received: 7 June 2018; Accepted: 7 June 2018; Published: 8 June 2018

Aerospace has launched annual awards to recognize outstanding papers published in the journal.

We are pleased to announce the first "Aerospace Best Paper Awards" for 2017. Nominations were chosen from all papers published in 2017, and decisions were made by the Editorial Board together with the Editorial Office. Following a review process by the Editorial Board, the following three top-voted research articles, in no particular order, have won "Aerospace Best Paper Awards" for 2017:

\section{Design and Performance of Modular 3-D Printed Solid-Propellant Rocket Airframes}

Rachel N. Hernandez, Harpreet Singh, Sherri L. Messimer and Albert E. Patterson

Aerospace 2017, 4, 17; doi:10.3390/aerospace4020017

Available online: http:/ /www.mdpi.com/2226-4310/4/2/17

Solid-propellant rockets are useful for many applications, including military technology, scientific research, entertainment, and aerospace education. While much research has been done in recent decades on liquid propellants and compatible rocket systems, small solid-propellant rockets are still unmatched for their low cost, reliability, and simplicity. One of the problems still encountered when designing rocket airframes for any application is the need to customize the airframe; however, this can be difficult since the production of rockets can be a complex and expensive process with many steps and interfaces between parts. A long and complex integration process is needed to reduce the reliability problems encountered at the various interfaces.

To begin to explore opportunities for easier and more efficient rocket airframe design, our group (Figure 1), led by Rachel N. Hernandez (now with NASA Kennedy Space Center, Florida, USA), developed a novel design modularization method for rocket airframes, utilizing additive manufacturing to combine multiple functions into a smaller number of parts. This method replaces part subsystems with complex multi-function parts to improve customization, design flexibility, performance, and reliability. For the simple rockets that were designed using our method, we were able to decrease the number of essential parts from 22 to four (engine, payload, nose cone, and engine mount) and were able to 3-D print all the parts except for the replaceable engines themselves.

To verify the value of this method, computational and experimental techniques were used to test a set of generated designs. The methods and the study results were examined in detail for sources of error, which could then be addressed on subsequent iterations of the design. A series of flights were performed on the designed rockets, with a total of eight launches. The method was found to be very successful in practice, with clear performance differences based on small modifications to the designs. A factorial designed experiment was used to analyze the flight results, providing even more detailed information about the value of a combination-of-functions modularization technique applied to the design of rocket airframes. The cost to produce the rockets was shown to be dramatically reduced (more than 50\%) using this technique as well. 


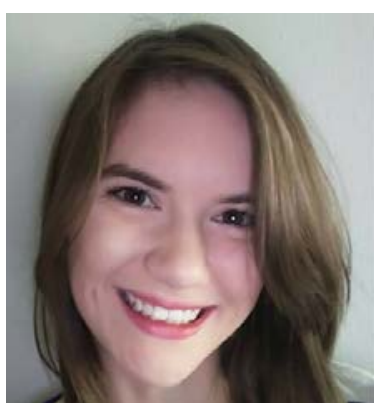

(a)

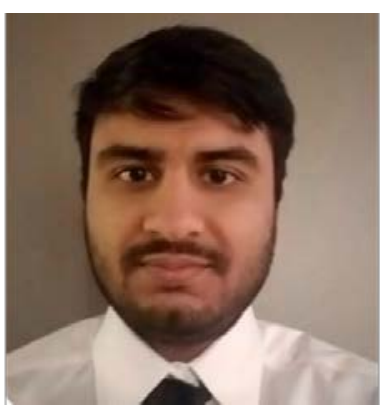

(b)

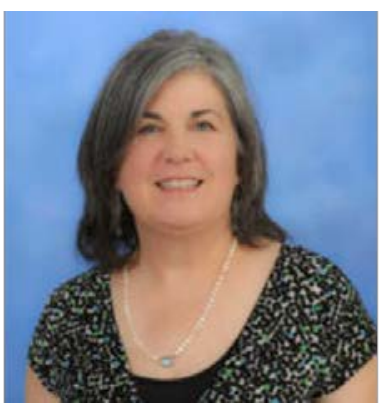

(c)

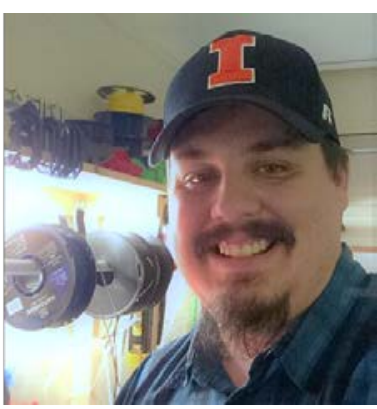

(d)

Figure 1. Mr. Patterson's research group.

\section{Stochastic Trajectory Generation Using Particle Swarm Optimization for Quadrotor Unmanned Aerial Vehicles (UAVs)}

Babak Salamat and Andrea M. Tonello

Aerospace 2017, 4, 27; doi:10.3390/aerospace 4020027

Available online: http:/ /www.mdpi.com/2226-4310/4/2/27

The paper "Stochastic Trajectory Generation Using Particle Swarm Optimization for Quadrotor Unmanned Aerial Vehicles (UAVs)", co-authored by Babak Salamat and Andrea M. Tonello (Figure 2), provides a realistic stochastic trajectory generation method for unmanned aerial vehicles. It offers a tool for the emulation of trajectories in typical UAV flight scenarios. In the domain of aerial robotics, there is a large body of literature on path planning and flight control. However, to assess performance, for instance of navigation algorithms, the trajectories followed by the moving aerial vehicle must be generated with a statistically representative emulator. In this paper, a new seminal idea on how to do so has been presented. In particular, three flying scenarios (flight level, takeoff-mission-landing, collision avoidance with complex maneuvering) are considered and defined in this paper. The trajectories for these scenarios are implemented with quintic B-splines, which grants smoothness in the second-order derivatives of the Euler angles and accelerations. In order to tune the parameters of the quintic B-spline in the search space, a multi-objective optimization method called particle swarm optimization (PSO) is used. The proposed technique satisfies the constraints imposed by the configuration of the UAV. Further constraints can be introduced such as: obstacle avoidance, speed limitation, and actuator torque limitations due to the practical feasibility of the trajectories. The results can open the door to novel methodologies for the development of stochastic trajectory generators.

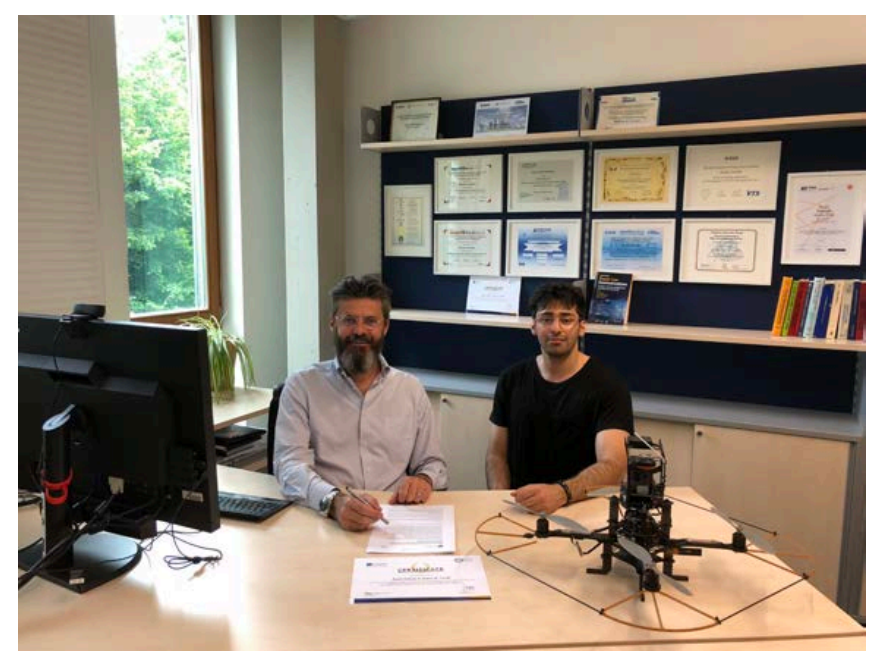

Figure 2. Prof. Tonello's research group. 


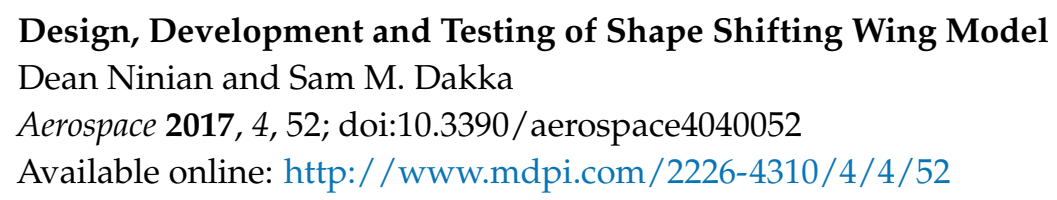

Conventional aircraft designs are becoming increasingly difficult to optimise for aerodynamic efficiency, therefore more radical and innovative technologies are required to tackle this problem. This research was completed to find the potential aerodynamic and noise reduction effects of morphing (shape shifting) wing technologies, co-authored by Dean Ninian and Sam M. Dakka (Figure 3).

The focus of the research is on the reducing lift-induced drag at the flaps of an aerofoil, then improve the design further to achieve maximum aerodynamic efficiency. Using a combination of simulation and conventional wind tunnel testing it was proven that the initial shape shifting wing achieved a $10.8 \%$ increase in coefficient of lift, and $15.4 \%$ increase for the optimised wing when compared to a conventional design. At angles of attack of 0, 5, 10 and 15 degrees, the lift-to-drag ratio increased by $18.3 \%, 10.5 \%, 10.6 \%$ and $4 \%$ respectively when compared to a conventional wing. Simulation showed that the optimised shape shifting wing had a $50 \%$ reduction in vortex size when compared to a conventional wing. This had a profound effect on the pressure distribution on the upper and lower surface of the wing, resulting in less pressure drag. The reduction in vortex size also resulted in a reduction in noise levels measured by up to $50 \%$.

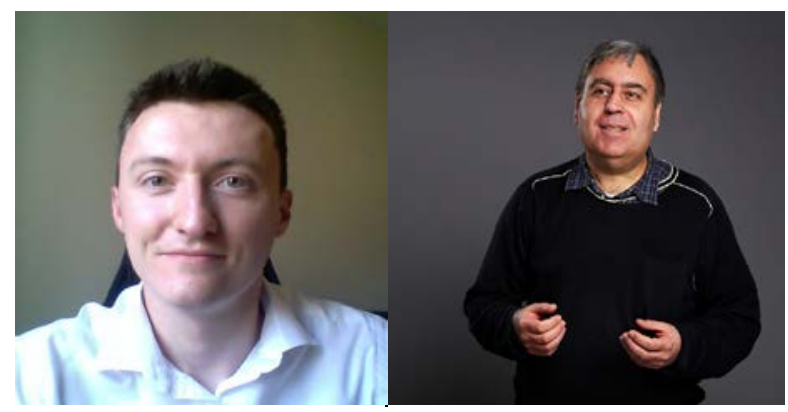

Figure 3. Dr. Dakka's research group.

We believe that these three exceptional papers are valuable contributions to Aerospace and the scientific research field. On behalf of the Aerospace Editorial Board, we would like to congratulate these teams for their excellent work. A certificate will be given to each of them.

We would like to take this opportunity to thank all the nominated research groups of the above exceptional papers for their contributions to Aerospace, and thank the Aerospace Editorial Board for voting and helping with these Best Paper Awards.

The Editorial Board and Editorial Staff at Aerospace is committed to meeting the needs of the scientific community by providing useful and timely reviews of all manuscripts submitted, and providing an open access forum for your results. Please consider submitting your work to Aerospace, and we look forward to announcing your paper as an Aerospace Best Paper in the future.

\section{Prize Awarding Committee}

Aerospace Editorial Board

(C) 2018 by the author. Licensee MDPI, Basel, Switzerland. This article is an open access article distributed under the terms and conditions of the Creative Commons Attribution (CC BY) license (http:/ / creativecommons.org/licenses/by/4.0/). 